\title{
Correlative Confocal and Light Microscopic Analysis of Rat Scalp Skin
}

\author{
Beverly E. Maleeff and Heath C. Thomas \\ Department of Safety Assessment, GlaxoSmithKline, King of Prussia, PA 19406
}

Drug-induced hair loss is a non-life-threatening condition that may affect the quality of life for patients. Treatments are currently being developed to prevent hair loss in this patient population. The neonatal rat was chosen as a suitable model because of its rapid hair growth and susceptibility to drug-induced hair loss [1]. In this study, we describe the appearance of rat scalp skin following topical application of two novel compounds with intrinsic fluorescent properties when excited with light at a wavelength of $351 \mathrm{~nm}$ [2]. This property acts as an internal fluorescent tag, allowing for cellular and tissue localization of these compounds after topical application. The purposes of this study were to determine the depth of compound penetration into the skin, localization of these compounds in neonatal rat scalp skin and hair, and the persistence of compounds over a time course.

Scalps of neonatal Sprague-Dawley rats, approximately 13 days old, were treated with a single topical application with one of two compounds, Compound A or Compound B, and samples were collected up to 96 hours later. Untreated scalp skin was collected as a control. Samples of skin for confocal laser scanning microscopic (CLSM) analysis were prepared by fixing briefly with $4 \%$ formaldehyde in phosphate buffered saline (PBS) and rinsing with PBS. For tissue localization, slices of skin, approximately $1 \mathrm{~mm}$ thick, were cut perpendicular to the epidermis and mounted cut side up between two coverslips in an Attofluor ${ }^{\circledR}$ cell chamber. Samples were examined using a Zeiss 510UV CLSM in confocal mode with excitation wavelengths of 351, 488 and $568 \mathrm{~nm}$ and the appropriate pinholes and emission filters, and in differential interference contrast (DIC) mode with the appropriate filters [3]. Wavelengths of light were chosen on the basis of spectrophotometric analysis of excitation and emission properties of both compounds (Figure 1) and available wavelengths of the CLSM lasers. For cellular localization, samples of skin for light microscopic examination were either unfixed or fixed as described above, cryoprotected with Tissue-Tek® OCT compound, snap-frozen in liquid nitrogen, and frozen sections approximately $6 \mu \mathrm{m}$ thick cut and mounted on microscope slides. Selected sections were stained with hematoxylin and eosin (H\&E) while others remained unstained; all were examined by transmitted light, CLSM and DIC.

In all cases, examination of untreated skin slices revealed no fluorescence after excitation with blue $(351 \mathrm{~nm})$ or green $(488 \mathrm{~nm})$ light (Figure 3). Red autofluorescence of hair shafts in control tissues was noted after excitation with light at $568 \mathrm{~nm}$. Scalp skin treated with either Compound A (Figure 4) or Compound B (data not shown) exhibited blue fluorescence only in perifollicular cells, i.e., compound. Identification of specific cell types was confirmed after examination of H\&Estained frozen sections (Figure 5, left); blue fluorescence was found in keratinized epithelium, nonkeratinized epithelium and adipocytes, but not within hair shafts (Figure 5, right). Additionally, the intensity of blue fluorescence in perifollicular cells was greatest after 2 hours of exposure to either compound and decreased by approximately $75 \%$ in tissues exposed to compound for 96 hours (Figure 6).

By determining the depth of penetration of the compounds into rat scalp skin, the cells into which the compounds localize, and the persistence of the compounds in the cells over time, we may be able to design appropriate clinical dosing paradigms for administration of these compounds to patients. 


\section{References}

[1] A.M. Hussein, et al., Science 249 (1990) 1564-1566.

[2] R. Brashear and D. Lu, personal communication.

[3] J.A. Veiro and P.G. Cummins, Clin. Lab. Invest. 189 (1994) 16-22.

[4] The authors gratefully acknowledge the contributions of D. Eberwein and N. Rhodes.

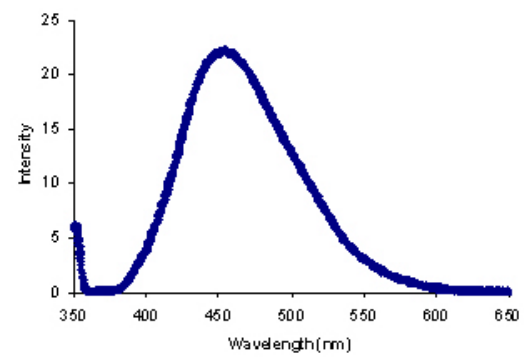

FIG. 1. Emission spectrum for Compound A after $351 \mathrm{~nm}$ excitation.
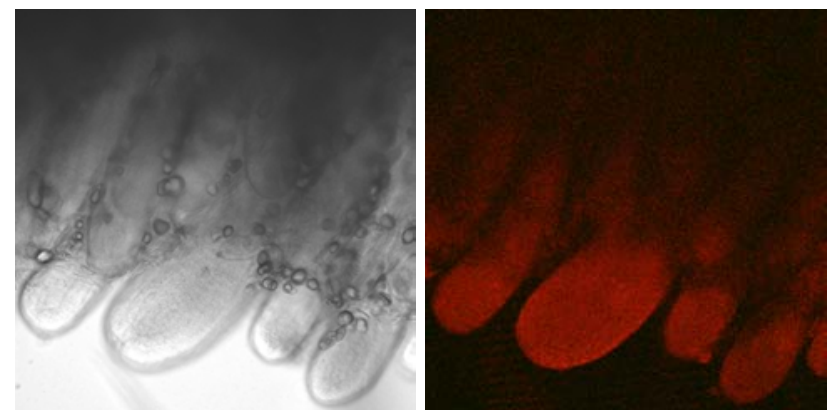

FIG. 3. DIC and fluorescence images of control scalp skin. Red emission is autofluorescence. Original magnification 20x.
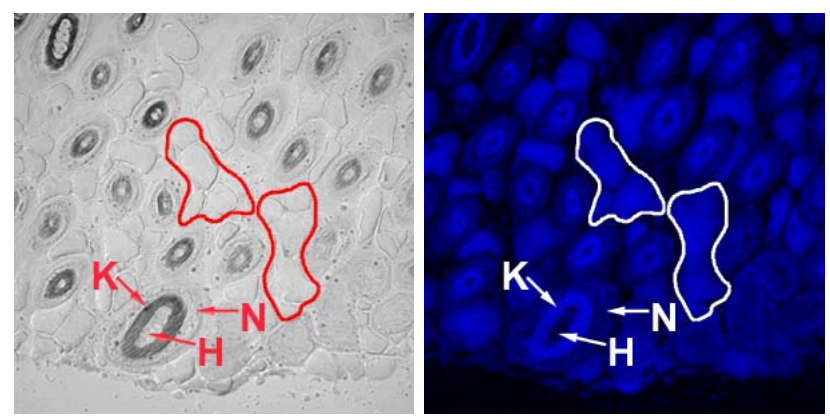

FIG. 5. DIC and fluorescence images of $6 \mu \mathrm{m}$ frozen sections of scalp skin 2 hours after exposure to Compound A. Left, H\&E. Right, fluorescence. Hair shaft $(\mathrm{H})$, keratinized $(\mathrm{K})$ and nonkeratinized $(\mathrm{N})$ epithelium, adipocytes (outlined). Original magnification 20x.

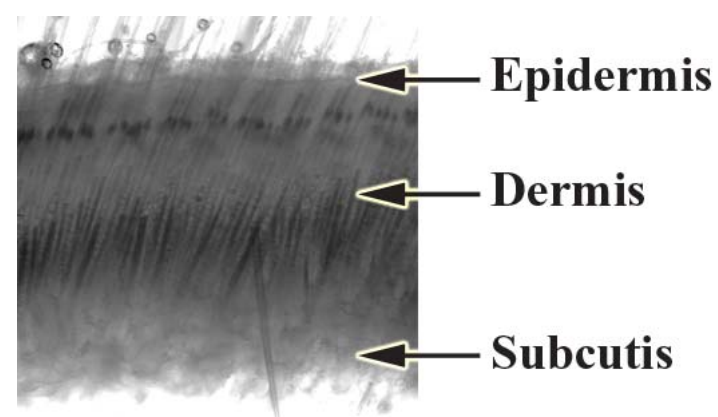

FIG. 2. DIC image of a $1 \mathrm{~mm}$ cross-section of neonatal rat scalp. Original magnification $5 x$.
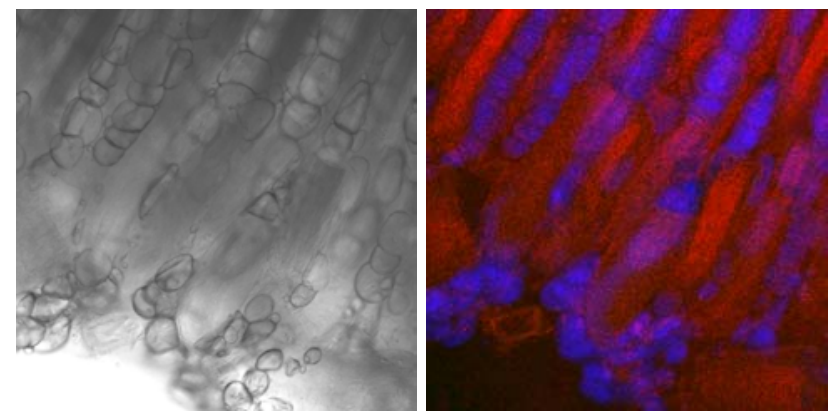

FIG. 4. DIC and fluorescence images of scalp skin exposed to Compound A.

Original magnification 20x.
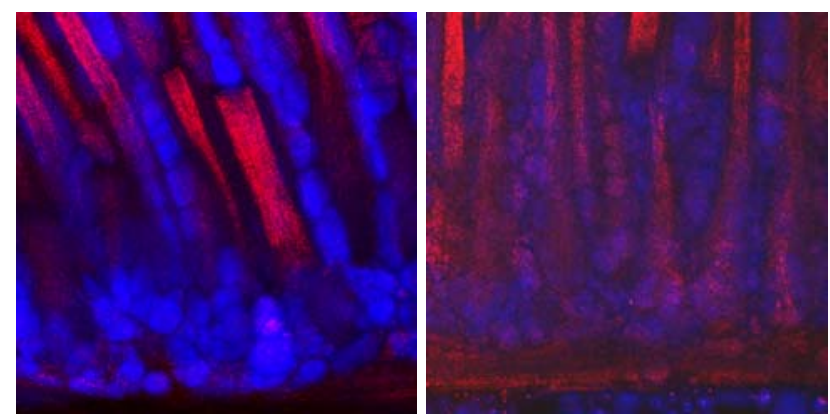

FIG. 6. Fluorescence images of scalp skin 2 hours and 96 hours after exposure to Compound A.

Original magnification 20x. 